\title{
Effect of hydrogen sulfide on restenosis of peripheral arteries after angioplasty
}

\author{
BINGBING MA ${ }^{1}$, GANGZHU LIANG ${ }^{1}$, FUXIAN ZHANG $^{1}$, YIZHI CHEN $^{2}$ and HUAN ZHANG ${ }^{1}$ \\ Departments of ${ }^{1}$ Vascular Surgery, and ${ }^{2}$ Pathology, Beijing Shijitan Hospital, \\ Ninth Clinical Hospital of Peking University, Haidian, Beijing 100038, P.R. China
}

Received December 13, 2011; Accepted March 26, 2012

DOI: $10.3892 / \mathrm{mmr} .2012 .853$

\begin{abstract}
Peripheral artery disease (PAD) may lead to a poor quality of life. Although percutaneous transluminal angioplasty (PTA) is widely used for the treatment of PAD, restenosis remains a major drawback. Hydrogen sulfide $\left(\mathrm{H}_{2} \mathrm{~S}\right)$ plays potential roles in many physiological processes, such as vasodilatation and inhibition of smooth muscle cell proliferation. However, little is known regarding its role in arterial restenosis. In this study, we induced atherosclerotic-like lesions in rabbits, and we treated the rabbits with balloon angioplasty (BA) in a similar manner as PTA performed in the clinic. The rabbits were treated with sodium hydrosulfide (NaHS, a donor of $\mathrm{H}_{2} \mathrm{~S}$ ) or DL-propargylglycine (PPG, an inhibitor of $\mathrm{H}_{2} \mathrm{~S}$ synthase). Treatment with NaHS significantly inhibited arterial restenosis following BA by reducing the intimal area and the intima/media ratio, while PPG treatment had a tendency to result in more severe restenosis. NaHS treatment significantly reduced smooth muscle cell (SMC) proliferation and elevated SMC apoptosis in the neointima. In contrast, PPG induced a significant increase in SMC proliferation. In conclusion, $\mathrm{H}_{2} \mathrm{~S}$ attenuates the progression of neointimal hyperplasia and inhibits restenosis after BA. This discovery may lead to potential novel therapies, which can improve the prognosis of PAD patients.
\end{abstract}

\section{Introduction}

Peripheral artery disease (PAD) mainly comprises atherosclerosis of the abdominal aorta, iliac and lower extremity arteries. Patients with PAD may encounter a multitude of problems, such as claudication, ischemic rest pain, ischemic ulcerations, repeated hospitalizations, revascularizations and limb loss.

Correspondence to: Professor Fuxian Zhang, Department of Vascular Surgery, Beijing Shijitan Hospital, Ninth Clinical Hospital of Peking University, Tie Yi Street No. 10, Haidian, Beijing 100038, P.R. China

E-mail: fuxianvascular@yahoo.cn

Key words: hydrogen sulfide, atherosclerosis, peripheral artery disease, percutaneous transluminal angioplasty, smooth muscle cell, neointimal hyperplasia, restenosis
These events may lead to a poor quality of life and a high rate of depression.

Although percutaneous transluminal angioplasty (PTA) is widely used for the treatment of PAD, restenosis remains a major drawback of this minimally invasive treatment, occurring in approximately $45.3 \%$ of procedures (1). Numerous studies attempting to increase vessel patency after PTA have been undertaken, but these studies have not met our expectations in preventing restenosis. The mechanisms of restenosis have not been completely elucidated, but vascular smooth muscle cell (SMC) proliferation has been considered to be a key event in the remodeling of the vessel wall following vascular restenosis after an invasive intervention (2).

Hydrogen sulfide $\left(\mathrm{H}_{2} \mathrm{~S}\right)$ has long been known as an environmental pollutant, but only recently has it been regarded as a novel gasotransmitter (3). Endogenous $\mathrm{H}_{2} \mathrm{~S}$ is generated from L-cysteine catalyzed by cystathionine- $\gamma$-lyase (CSE) in the cardiovascular system. It has potential roles in many physiological processes and disease states in the human body and exerts regulatory effects on the pathogenesis of various cardiovascular diseases such as hypertension, pulmonary hypertension, shock and myocardial injury (4-6). A significant decrease in plasma $\mathrm{H}_{2} \mathrm{~S}$ was observed in patients with coronary heart disease, and plasma $\mathrm{H}_{2} \mathrm{~S}$ levels have been correlated with the severity of coronary heart disease (7). Furthermore, the vascular $\mathrm{CSE} / \mathrm{H}_{2} \mathrm{~S}$ pathway was found to be disturbed in the pathogenesis of atherosclerosis (8). However, the role of $\mathrm{H}_{2} \mathrm{~S}$ in the pathogenesis of restenosis following PTA for the treatment of PAD is incompletely understood. This study was designed to observe $\mathrm{H}_{2} \mathrm{~S}$ expression after PTA and the influence of $\mathrm{H}_{2} \mathrm{~S}$ inhibition on restenosis following PTA in a rabbit atherosclerotic model.

\section{Materials and methods}

Ethics statement. All animal care and procedures conformed with the Guide for the Care and Use of Laboratory Animals published by the US National Institutes of Health (NIH Publication no. 85-23, revised 1996) and were approved by Peking University Institutional Animal Care and Use Committee (Permit numbers 2008-017).

Materials. Sodium hydrosulfide (NaHS), DL-propargylglycine (PPG), hematoxylin and pyridoxal 5'-phosphate were 
purchased from Sigma-Aldrich (St. Louis, MO, USA). TRIzol reagent was obtained from Invitrogen (Carlsbad, CA, USA). dNTP mix, oligo(dT)15 primer and M-MLV reverse transcriptase were purchased from Promega (Madison, WI, USA). SYBR Green PCR Master Mix was purchased from Applied Biosystems (Foster, CA, USA). All other chemicals were of analytical grade and were purchased from Beijing Chemical Reagents (Beijing, China).

Experimental design. Two sequential series of experiments were performed. The first phase examined the alteration of the vascular $\mathrm{CSE} / \mathrm{H}_{2} \mathrm{~S}$ pathway following double injury of the femoral arteries of New Zealand white rabbits, and the second, examined the effects of $\mathrm{H}_{2} \mathrm{~S}$ donor therapy on restenosis.

Rabbits were randomly divided into four groups depending on treatment: normal control $(n=8)$, model control $(n=16)$, NaHS ( $\mathrm{n}=16 ; \mathrm{H}_{2} \mathrm{~S}$ donor at $56 \mu \mathrm{mol} / \mathrm{kg}$ body weight/day) and PPG ( $\mathrm{n}=16$; CSE inhibitor at $37.5 \mathrm{mg} / \mathrm{kg}$ body weight/day). NaHS and PPG were freshly prepared each day, dissolved in physiologic saline and injected intraperitoneally into rabbits. Untreated rabbits were injected daily with saline (9). The three experimental groups of rabbits presented with atherosclerosis and received balloon angioplasty (BA), then were treated as mentioned above, immediately before BA and every day after BA until the end of the experiment. The three experimental groups of rabbits were euthanized at 1 or 4 weeks (10) after BA ( $\mathrm{n}=8$, respectively).

Animal model. Male New Zealand white rabbits (weighing 3.5-4 kg) were obtained from the Laboratory Animal Center of The Academy of Military Medical Sciences (Beijing, China) and raised in the Experimental Animal Center of Peking University.

Atherosclerosis was induced in the femoral arteries of New Zealand white rabbits by a combination of air desiccation and a high-cholesterol diet as previously described $(11,12)$. Briefly, rabbits were anesthetized by an intramuscular injection of xylazine $(5 \mathrm{mg} / \mathrm{kg})$ and ketamine $(35 \mathrm{mg} / \mathrm{kg})$. Proximal and distal ligatures were used to isolate $1-\mathrm{cm}$-long sections of the femoral arteries of the rabbits. The isolated segments were cannulated with a 27 -gauge needle, and a vent was created by needle puncture; the segments were desiccated by air infused at a rate of $120 \mathrm{ml} / \mathrm{min}$ for $15 \mathrm{~min}$. Following air drying, the isolated segments were flushed with saline, and the ligature was removed. The desiccated segments were marked at the body surface. The day following the surgery, the animals were fed a $2 \%$ cholesterol and $6 \%$ peanut oil diet for 4 weeks.

Four weeks after the induction of atherosclerosis, BA was performed as previously described with minor modifications $(11,12)$. Briefly, the animals were again anesthetized with xylazine and ketamine. The superficial femoral artery was isolated below the knee joint, and the distal end was ligated. A 5F introducer was placed via an arteriotomy. Heparin $(150 \mathrm{U} / \mathrm{kg})$ and lidocaine $(20 \mathrm{mg})$ were injected intra-arterially. According to the body surface marker, a $2.5-\mathrm{mm}$ balloon catheter was positioned across the stenosis in the femoral artery. The balloon was inflated to $5 \mathrm{~atm}$ for $60 \mathrm{sec}$, and this was repeated three times at 60 -sec intervals. The catheter was then removed, the superficial femoral artery was ligated, and the profound femoral artery was left for runoff. The same procedure was repeated in the contralateral femoral artery. Thereafter, rabbits had free access to normal food and water and were observed for 4 weeks.

Tissue processing. One or four weeks after angioplasty, the animals were euthanized by an overdose of pentobarbital sodium $(120 \mathrm{mg} / \mathrm{kg})$. The femoral arteries were perfused with phosphate-buffered saline (PBS, $0.01 \mathrm{~mol} / \mathrm{l}, \mathrm{pH} 7.4$ ) and immediately perfusion-fixed at a constant physiological pressure of $110 \mathrm{mmHg}$ with $4 \%$ phosphate-buffered paraformaldehyde or removed and stored at $-80^{\circ} \mathrm{C}$.

Measurement of $\mathrm{H}_{2} \mathrm{~S}$ content in plasma. Plasma $\mathrm{H}_{2} \mathrm{~S}$ was measured in duplicate samples with a sulfide electrode (PXS-270, Shanghai, China) (9). Briefly, $0.5 \mathrm{ml}$ of plasma was mixed with $0.5 \mathrm{ml}$ of antioxidant buffer. After being rinsed with deionized water and dried, the electrode was immersed into the sample. The electrode potential was recorded when the reading stabilized. The $\mathrm{H}_{2} \mathrm{~S}$ concentration was calculated by comparison to a standard curve.

Measurement of $\mathrm{H}_{2} \mathrm{~S}$ production in tissues. The $\mathrm{H}_{2} \mathrm{~S}$ production rate was measured to reflect CSE activity as described previously (9). Briefly, femoral arteries were homogenized in $1: 10(\mathrm{w} / \mathrm{v}) 50 \mathrm{mmol} / \mathrm{l}$ ice-cold potassium phosphate buffer (pH 6.8). Reactions were performed in a $25-\mathrm{ml}$ Erlenmeyer flask. The reaction mixtures contained $10 \mathrm{mmol} / \mathrm{l} \mathrm{L}$-cysteine, $2 \mathrm{mmol} / \mathrm{l}$ pyridoxal 5'-phosphate, $100 \mathrm{mmol} / \mathrm{l}$ potassium phosphate buffer ( $\mathrm{pH} 7.4)$ and $10 \%(\mathrm{w} / \mathrm{v})$ homogenates. Cryovial test tubes $(2 \mathrm{ml})$ were used as the center wells, and each contained $0.5 \mathrm{ml}$ of $1 \mathrm{~mol} / 1$ sodium hydroxide. The flask was then flushed with $\mathrm{N}_{2}$ before being sealed with parafilm. After incubation at $37^{\circ} \mathrm{C}$ for $90 \mathrm{~min}, 0.5 \mathrm{ml}$ of $50 \%$ trichloroacetic acid were added to the mixture to terminate the reaction. The flasks were incubated in a shaking water bath for an additional $60 \mathrm{~min}$ at $37^{\circ} \mathrm{C}$ to ensure complete trapping of $\mathrm{H}_{2} \mathrm{~S}$. The contents of the center well were transferred to test tubes and mixed with $0.5 \mathrm{ml}$ of antioxidant buffer. The $\mathrm{H}_{2} \mathrm{~S}$ content in the solution was also measured with a sulfide electrode in a way identical to that of the measurement in plasma. The measurement was performed in duplicate for each sample. The $\mathrm{H}_{2} \mathrm{~S}$ production rate was expressed in $\mathrm{nmol} /(\min \mathrm{x} \mathrm{mg}$ protein).

Quantitation of CSE $m R N A$ level by real-time polymerase chain reaction $(P C R)$. Total RNA was extracted from the femoral arteries using TRIzol reagent and transcribed into cDNA using oligo(dT)15 primer and M-MLV reverse transcriptase. Quantitative RT-PCR was performed with an ABI PRISM 7900HT instrument (Applied Biosystems) using SYBR Green PCR Master Mix. For quantification, the target gene was normalized by comparison to the internal standard gene $\beta$-actin. The primers of CSE (GenBank accession number XM_002715559) were 5'-CCGAGAAGTCCTTGGGATCA-3' (sense primer) and 5'-CTGCCTTCAAAGCCTGGTCTA-3' (antisense primer). Relative mRNA quantification was calculated with the arithmetic formula $2^{-\Delta \Delta C T}(13)$, where $\Delta \mathrm{CT}$ is the difference between the threshold cycle of a given target cDNA and an endogenous reference $\beta$-actin cDNA. Based on the calculated $\triangle \mathrm{CT}$ value, the CSE mRNA level in the target 
femoral arteries was subsequently expressed as the percentage of that in the normal controls.

Morphometric analyses. The femoral arteries were perfusionfixed at a constant physiological pressure of $110 \mathrm{mmHg}$ with $4 \%$ phosphate-buffered paraformaldehyde. Then, they were carefully stripped of extravascular tissue, and excised between the origin at the iliac artery and the femoral bifurcation. The double-injured segment of the denuded arteries was removed and fixed in $4 \%$ paraformaldehyde for $12 \mathrm{~h}$. Each femoral artery was cut serially at 1 - to $2-\mathrm{mm}$ intervals from the proximal to the distal end and embedded in paraffin. The cross sections $(5 \mu \mathrm{m})$ in femoral arteries were stained with hematoxylin and eosin and then stained with Verhoeff's elastic stain to clearly delineate the internal and external elastic lamina. Morphometric analysis of the arterial segments was carried out by an observer blinded to the study groups using computerized digital microscopic planimetry software (Image-Pro Plus, Version 6.0 for Windows, Media Cybernetics, Silver Spring, MD, USA). The lesion site was defined by the cross section with the smallest luminal area of the serial sections. The crosssection areas subtended by the luminal border, the internal elastic lamina (IEL), and the external elastic lamina (EEL) were measured, and the ratio of intimal and medial areas (I/M) was calculated (14).

Immunohistological staining for PCNA and TUNEL in the femoral arteries. Proliferation was assessed by proliferating cell nuclear antigens (PCNAs) (15). Sections (5 $\mu \mathrm{m})$ were deparaffinized, rehydrated, and treated with a $10 \mathrm{mM}$ sodium citrate buffer (pH 6.0; boiling for $20 \mathrm{~min}$ ) to uncover masked antigens. Then endogenous peroxidase was blocked with $3 \% \mathrm{H}_{2} \mathrm{O}_{2}$ for $10 \mathrm{~min}$ at room temperature. After sections were blocked with normal horse serum for $20 \mathrm{~min}$, a specific primary antibody, anti-PCNA antibody (PC10; Dako Corp., Carpinteria, CA, USA; 1:1000), was applied overnight at $4^{\circ} \mathrm{C}$. The secondary antibody incubation (biotinylated anti-mouse antibody, dilution 1:1000) was for $20 \mathrm{~min}$ at $37^{\circ} \mathrm{C}$, followed by amplification with an avidin-biotin complex (ABC) reagent (Vector Laboratories, Burlingame, CA, USA) for 30 min. Incubation with 3',3'-diaminobenzidine (DAB) produced a brown reaction product. Hematoxylin was used for counterstaining. Brown granules under microscopy were defined as PCNA-positive signals.

Apoptosis was assessed by the terminal deoxynucleotidyl transferase dUTP nick-end labeling (TUNEL) method using a TUNEL apoptosis detection kit (catalog no. 11684817910; Roche, Basel, Switzerland) following the procedure in the manufacturer's instructions. In brief, $5-\mu \mathrm{m}$ sections were deparaffinized and incubated with proteinase K [1/24 (v/v) in phosphate-buffered saline] for $15 \mathrm{~min}$ at room temperature. Sections were incubated with the terminal deoxynucleotidyl transferase end-labeling cocktail (a mixture of terminal deoxynucleotidyl transferase buffer, biotin-dUTP, and terminal deoxynucleotidyl transferase at a ratio of 90:5:5, respectively) for $60 \mathrm{~min}$ at $37^{\circ} \mathrm{C}$. After washing and blocking, avidinfluorescein isothiocyanate (1:10) was applied to the sections, which were then incubated in the dark for $30 \mathrm{~min}$ at $37^{\circ} \mathrm{C}$.

Quantification of apoptosis and cell proliferation. Apoptosis and cell proliferation were identified by computerized digital
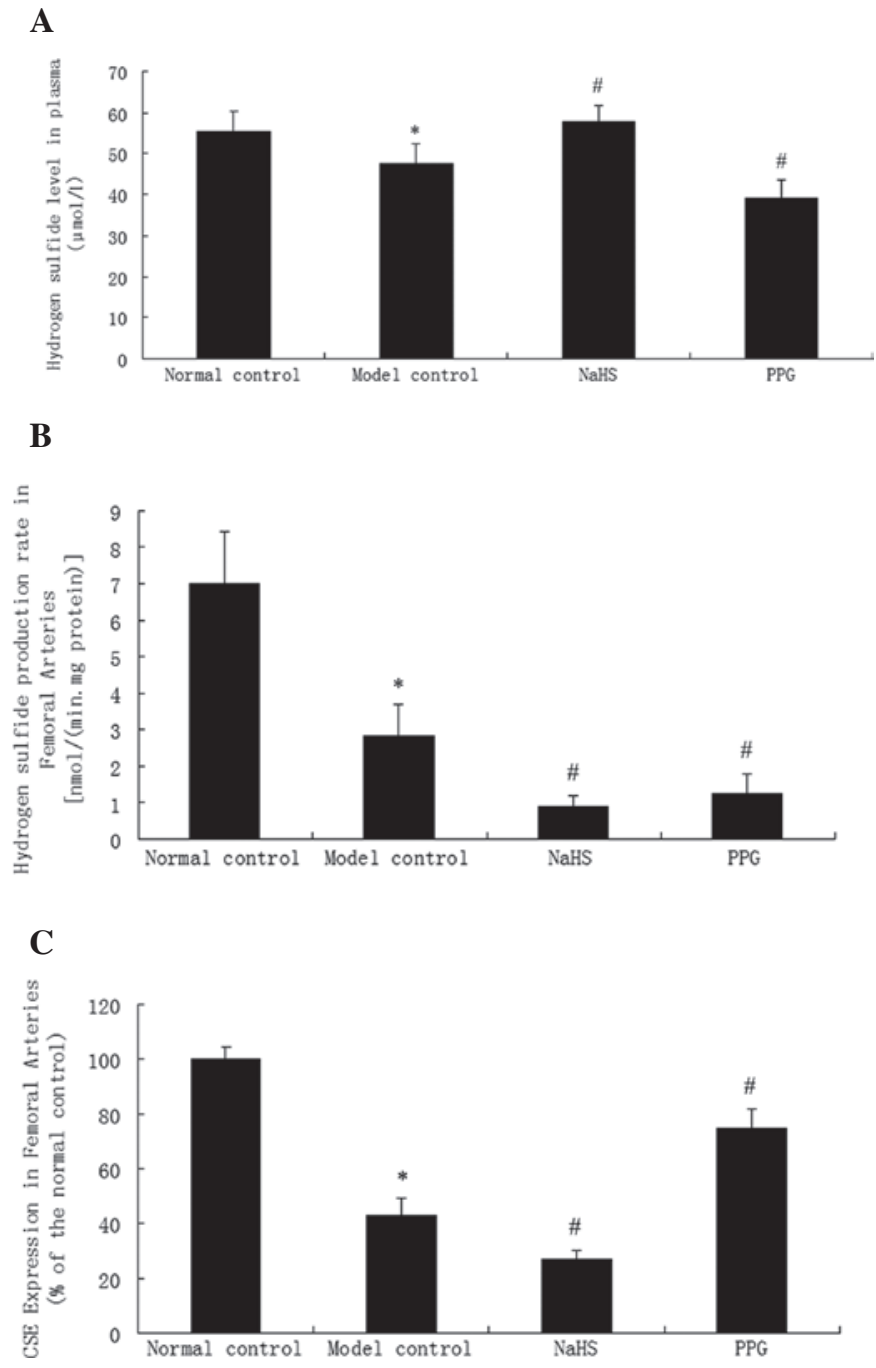

Figure 1. Changes in the $\mathrm{CSE} / \mathrm{H}_{2} \mathrm{~S}$ pathway (mean $\pm \mathrm{SD}$ ). (A) $\mathrm{H}_{2} \mathrm{~S}$ levels in plasma at the end of the experiment. (B) Endogenous production of $\mathrm{H}_{2} \mathrm{~S}$ in femoral arteries. (C) Expression of CSE mRNA in femoral arteries. ${ }^{*} \mathrm{P}<0.01$ compared to the normal controls. ${ }^{\#} \mathrm{P}<0.01$ compared to the model controls. PPG, DL-propargylglycine.

microscopic planimetry software in the neointima, media and adventitia. This analysis was conducted by an observer blinded to the study groups. Apoptotic cells were quantified by counting the percentage of TUNEL-positive cells against the total number of nucleated cells in 12 different fields per tissue section, and the ratio was expressed as the apoptosis index. Cell proliferation was determined by the proliferation index expressed as the ratio of PCNA-positive cells to total nucleated cells. A total of 12 different fields from each artery section were randomly selected, and the numbers of PCNA-positive and total nuclei were counted.

Statistical analysis. Data analysis involved the use of SPSS v13.0 for Windows (SPSS Incorporated, Chicago, IL, USA). The results are expressed as the means \pm standard deviation (SD). Comparisons among groups involved one-way ANOVA followed by the LSD test, while the two-sample t-test was used for comparisons between two groups. $\mathrm{P}<0.05$ was considered to indicate a statistically significant difference. 


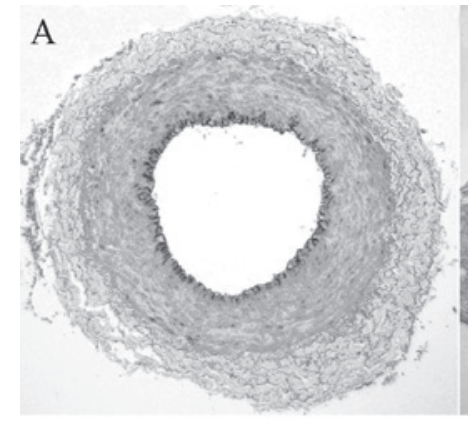

$\mathbf{E}$

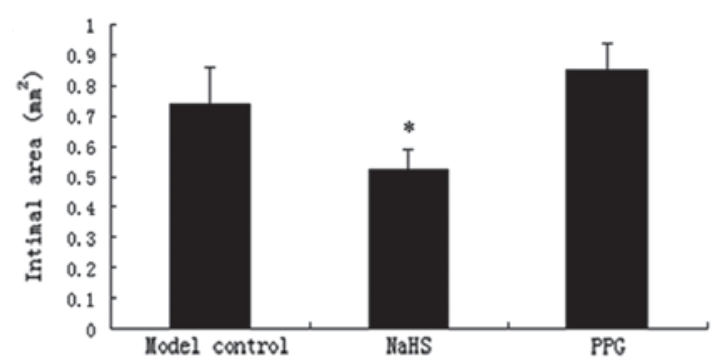

G

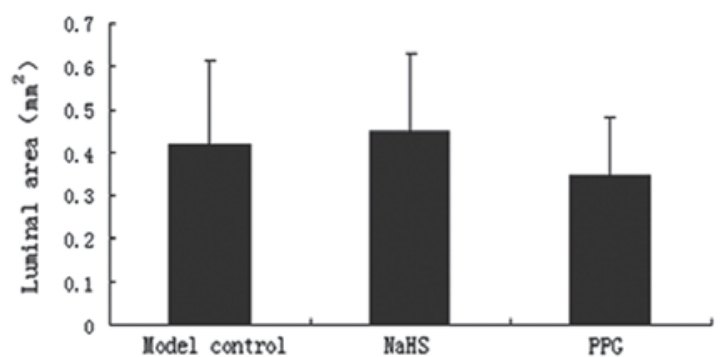

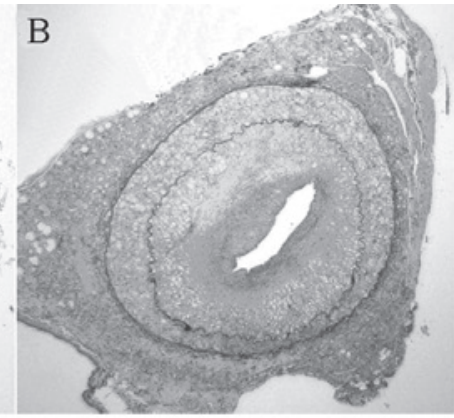

$\mathrm{C}+\mathrm{D}$

D

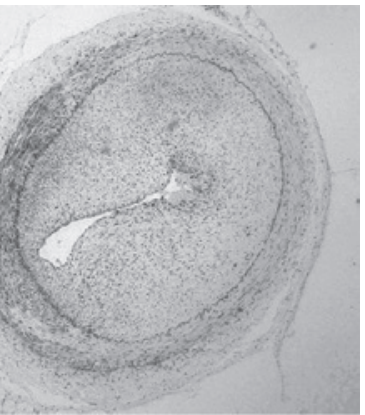

$\mathbf{F}$

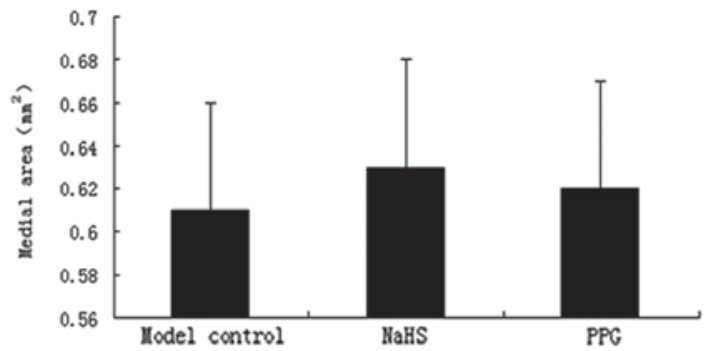

$\mathbf{H}$

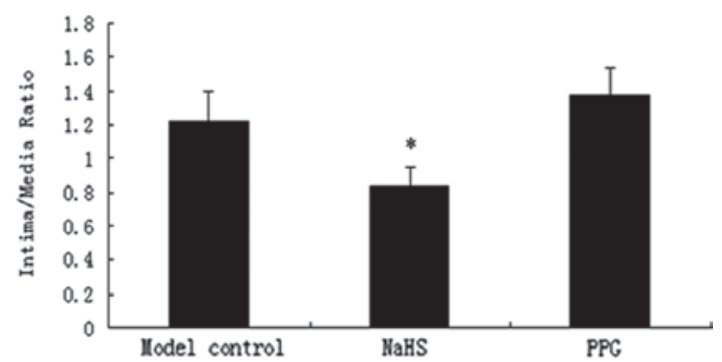

Figure 2. Histological and morphometric analyses of rabbit femoral arteries. (A) Representative of normal femoral arteries. (B) Representative of morphological changes in femoral arteries 4 weeks after balloon angioplasty (BA), showing severe neointimal hyperplasia. (C) Representative of morphological changes in femoral arteries 4 weeks after BA and receiving sodium hydrosulfide (NaHS), showing significant inhibition of neointima formation compared with B. (D) Representative of morphological changes in femoral arteries 4 weeks after BA and receiving DL-propargylglycine (PPG), showing no significant difference from B (Verhoeff's elastic stain). Original magnifications x40. (E) Morphometric analysis of the intima, (F) media, (G) luminal area and (H) intima/media ratio in femoral arteries. " $\mathrm{P}<0.01$ compared to the model controls.

\section{Results}

$\mathrm{H}_{2} \mathrm{~S}$ levels in plasma. Plasma $\mathrm{H}_{2} \mathrm{~S}$ levels were measured 4 weeks after BA (Fig. 1A). The model control rabbits showed a significantly lower $\mathrm{H}_{2} \mathrm{~S}$ level in plasma $(47.31 \pm 5.31$ vs. $55.47 \pm 4.87$ $\mu \mathrm{mol} / 1, \mathrm{P}<0.01)$ compared to the normal control rabbits. In $\mathrm{NaHS}$ rabbits, plasma $\mathrm{H}_{2} \mathrm{~S}$ was significantly increased $(57.87 \pm 3.75 \mu \mathrm{mol} / 1, \mathrm{P}<0.01)$ compared to the model control rabbits. In the PPG rabbits, plasma $\mathrm{H}_{2} \mathrm{~S}(39.21 \pm 4.40 \mu \mathrm{mol} / 1$, $\mathrm{P}<0.01)$ was significantly decreased compared to the model control rabbits.

Endogenous production of $\mathrm{H}_{2} \mathrm{~S}$ in femoral arteries. Compared to the normal controls, the model control rabbits showed significantly lower femoral production of $\mathrm{H}_{2} \mathrm{~S}[2.84 \pm 0.87$ vs. $7.02 \pm 1.40 \mathrm{nmol} /(\mathrm{min} \times \mathrm{mg}$ protein $), \mathrm{P}<0.01]$; in NaHS and PPG rabbits, the production rates of $\mathrm{H}_{2} \mathrm{~S}$ in the femoral arteries were still significantly decreased $[0.86 \pm 0.31 \mathrm{nmol} /(\min \mathrm{x} \mathrm{mg}$ protein), $\mathrm{P}<0.01 ; 1.24 \pm 0.57 \mathrm{nmol} /(\min \mathrm{x}$ mg protein), $\mathrm{P}<0.01$, respectively] compared to the model control rabbits (Fig. 1B).

Expression of CSE MRNA in femoral arteries. CSE expression in the femoral artery was determined by real-time PCR and normalized to $\beta$-actin levels. In the model controls, CSE
mRNA was significantly suppressed to $42.6 \pm 6.6 \%(\mathrm{P}<0.01)$, considering the expression of normal controls as $100 \%$. NaHS treatment further decreased the expression of CSE mRNA $(26.8 \pm 3.2 \%, \mathrm{P}<0.01)$ compared to the model controls, whereas PPG treatment significantly increased the expression of CSE mRNA (74.7 $\pm 7.2 \%, \mathrm{P}<0.01$; Fig. 1C).

Inhibitory effects of $\mathrm{H}_{2} \mathrm{~S}$ on restenosis in femoral arteries after balloon angioplasty (BA). Development of restenosis after BA was confirmed (Fig. 2A-D). Compared to the model controls, treatment with NaHS significantly inhibited neointima formation $\left(0.52 \pm 0.07\right.$ vs. $0.74 \pm 0.12 \mathrm{~mm}^{2}, \mathrm{P}<0.01$; Fig. $\left.2 \mathrm{E}\right)$ and reduced the intima/media ratios $(0.84 \pm 0.11$ vs. $1.22 \pm 0.18$, $\mathrm{P}<0.01$ ) of the femoral arteries (Fig. $2 \mathrm{H}$ ), but there were no significant differences in the medial and luminal areas of the femoral arteries (Fig. 2F and G). Furthermore, there were no differences between the PPG rabbits and the model controls in any measurement of restenosis. Luminal areas were the same in the two groups, as well as the neointimal and medial areas and intima/media ratios (Fig. 2E-H).

Anti-proliferative and apoptotic effects of $\mathrm{H}_{2} \mathrm{~S}$ on vascular smooth muscle cells (SMCs). SMC proliferation and apoptosis were determined 1 week after BA. Compared to the model 

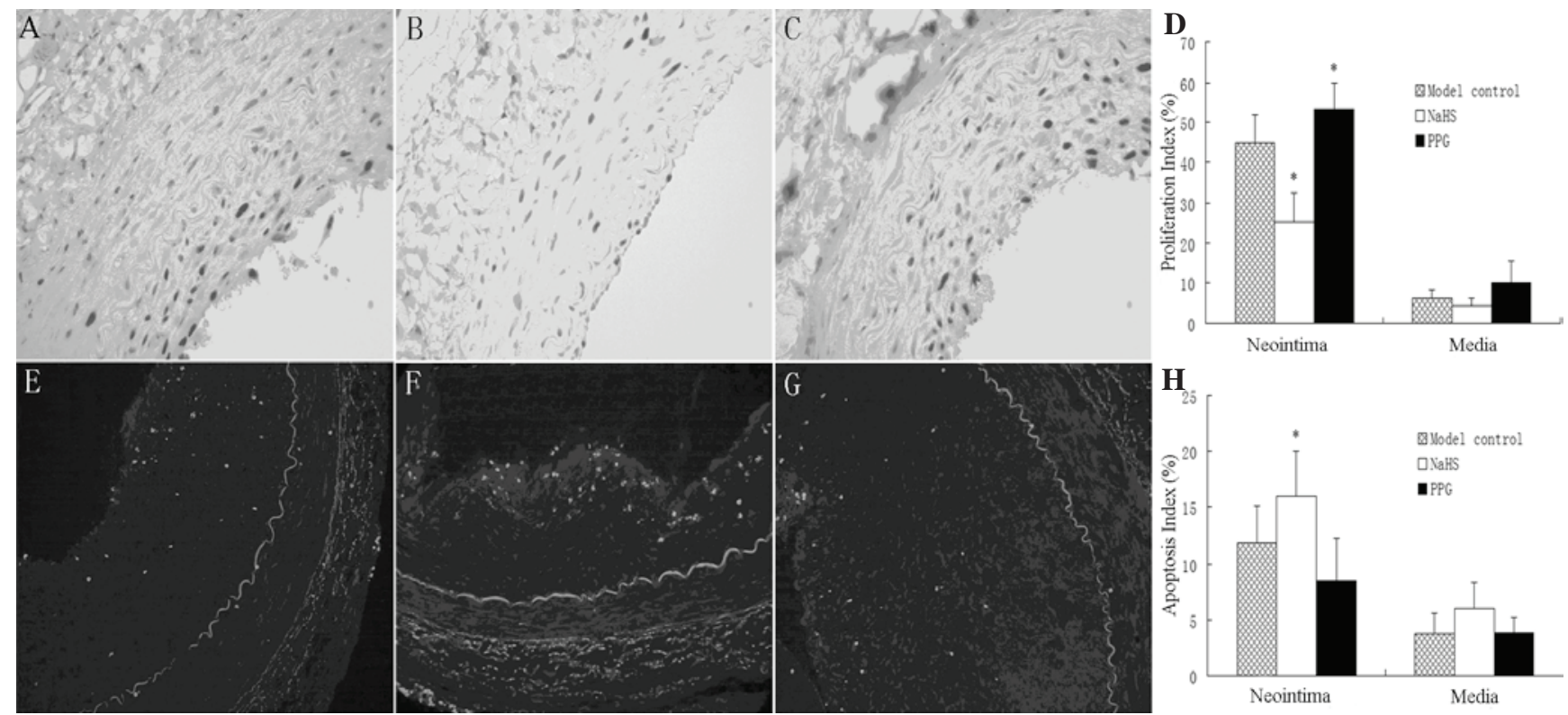

Figure 3. Immunohistochemistry of proliferating cell nuclear antigen (PCNA) expression and TUNEL staining in femoral arteries. (A and E) Representative of model control arteries. (B and F) Representative of sodium hydrosulfide (NaHS)-treated arteries. (C and G) Representative of DL-propargylglycine (PPG)-treated arteries. Original magnification, x200. (D and H) Proliferation and apoptosis indices in the femoral arteries. ${ }^{*} \mathrm{P}<0.01$ compared to the model controls.

controls, treatment with NaHS induced a significant reduction in the percentage of PCNA-positive cells in the neointima $(25.3 \pm 7.0$ vs. $45.1 \pm 6.8 \%, \mathrm{P}<0.01)$, whereas $\mathrm{PPG}$ increased proliferation in the neointima $(53.5 \pm 6.5$ vs. $45.1 \pm 6.8 \%$, $\mathrm{P}<0.01)$. Neither NaHS nor PPG induced significant differences of PCNA-positive cells in the media area compared to the model controls (Fig. 3A-D). TUNEL staining indicated that NaHS increased apoptosis in the neointima compared to the model controls $(16.0 \pm 4.0$ vs. $11.9 \pm 3.2 \%, \mathrm{P}<0.01)$, but the difference was not significant in the media area $(5.9 \pm 2.4$ vs. $3.7 \pm 1.8 \%, \mathrm{P}>0.05)$. There were no differences between the PPG rabbits and the model controls in SMC apoptosis (Fig. 3E-H).

\section{Discussion}

In previous research, the level of endogenous $\mathrm{H}_{2} \mathrm{~S}$ in atherosclerotic mice was found to be significantly reduced (8). After we treated the atherosclerotic femoral arteries with BA, we demonstrated that the $\mathrm{H}_{2} \mathrm{~S}$ levels in plasma, and CSE expression and $\mathrm{H}_{2} \mathrm{~S}$ production rates in the target femoral arteries were still significantly lower compared to the normal controls. Our findings suggest that CSE expression is downregulated and that its activity is suppressed during the development of atherosclerosis, and these responses were not corrected after BA. Together with the increasing neointimal hyperplasia, the $\mathrm{CSE} / \mathrm{H}_{2} \mathrm{~S}$ pathway is likely involved in restenosis progression after BA. That is, the low level of $\mathrm{H}_{2} \mathrm{~S}$ observed during neointimal hyperplasia indicates that $\mathrm{H}_{2} \mathrm{~S}$ may be protective against restenosis. Thus, we treated the rabbits with NaHS or PPG to explore the role of endogenous $\mathrm{H}_{2} \mathrm{~S}$ in restenosis pathogenesis. NaHS was used as a $\mathrm{H}_{2} \mathrm{~S}$ donor, and PPG, a potent, irreversible inhibitor of CSE, was used as an inhibitor of $\mathrm{H}_{2} \mathrm{~S}$ production $(9,16)$.

NaHS treatment significantly elevated the $\mathrm{H}_{2} \mathrm{~S}$ levels in plasma but reduced endogenous production of $\mathrm{H}_{2} \mathrm{~S}$ and the
CSE mRNA expression in the femoral arteries of the NaHS rabbits, whereas PPG significantly reduced the $\mathrm{H}_{2} \mathrm{~S}$ levels in plasma and $\mathrm{H}_{2} \mathrm{~S}$ production in the femoral arteries but elevated the CSE mRNA expression. These results suggest the possibility of negative feedback between circulating $\mathrm{H}_{2} \mathrm{~S}$ and CSE gene expression. The elevation of plasma $\mathrm{H}_{2} \mathrm{~S}$ in the NaHS rabbits resulted from the production of $\mathrm{H}_{2} \mathrm{~S}$ donor - NaHS. The reduction of plasma $\mathrm{H}_{2} \mathrm{~S}$ in the PPG rabbits was probably attributable to the inhibition of CSE in the femoral arteries.

The degree of restenosis in the femoral arteries, as mainly determined by the intimal area and intima/media ratio, was significantly reduced in the rabbits receiving NaHS but was more severe with PPG treatment compared to the model controls. This finding demonstrates that administration of $\mathrm{H}_{2} \mathrm{~S}$ inhibits neointima hyperplasia, thus $\mathrm{H}_{2} \mathrm{~S}$ may have a protective effect against restenosis.

In the present study, our team investigated the underlying mechanisms of the protective effect of $\mathrm{H}_{2} \mathrm{~S}$ on the development of neointimal hyperplasia. Compared to the model controls, NaHS treatment significantly reduced SMC proliferation in the neointima, whereas PPG induced a significant increase in the percentage of PCNA-positive cells in the neointima. Using TUNEL staining, we found that $\mathrm{H}_{2} \mathrm{~S}$ induced SMC apoptosis in the femoral arteries. These anti-proliferative and pro-apoptotic effects may explain the inhibitory effect of $\mathrm{H}_{2} \mathrm{~S}$ on restenosis after BA.

In previous studies, Yang et al (17) found that the antiproliferative effect of $\mathrm{H}_{2} \mathrm{~S}$ is mediated via ERK and $\mathrm{p} 21^{\text {Cip/WAK-1 }}$ activation. Yang et al (18) also demonstrated that both endogenous and exogenous $\mathrm{H}_{2} \mathrm{~S}$ could induce apoptosis of human aortic SMCs by upregulating caspase 3 and p21 ${ }^{\text {Cip/WAK-1 }}$ through activation of ERK and p38 MAPK. Furthermore, Baskar et al (19) found that asynchronized SMCs treated with an $\mathrm{H}_{2} \mathrm{~S}$ donor showed an increase in the number of apoptotic cells.

In addition, $\mathrm{H}_{2} \mathrm{~S}$ was found to attenuate expression of ICAM-1 via suppression of NF- $\kappa$ B activation (8). ICAM-1 
plays an important role in mediating the adhesion of inflammatory cells to the endothelium as well as the transmigration of leukocytes. The inflammatory process mediated by cellular adhesion molecules is involved in the occurrence and development of atherosclerosis $(20,21)$. Therefore, $\mathrm{H}_{2} \mathrm{~S}$ may inhibit restenosis by suppressing ICAM-1.

In the present study, a rabbit model of air-desiccationinduced atherosclerosis was used to simulate the natural course of peripheral arterial atherosclerotic disease. After performing balloon dilation, we investigated for the first time the CSE/ $\mathrm{H}_{2} \mathrm{~S}$ pathway and its regulatory role in the development of restenosis. CSE expression was reduced during the process of restenosis after BA. Reduced CSE expression led to decreased $\mathrm{H}_{2} \mathrm{~S}$ production and $\mathrm{H}_{2} \mathrm{~S}$ levels in plasma. Treatment with $\mathrm{H}_{2} \mathrm{~S}$ attenuated the progression of neointimal hyperplasia. The anti-proliferative and pro-apoptotic effects of $\mathrm{H}_{2} \mathrm{~S}$ on SMCs are possibly the mechanisms of the inhibitory effect of $\mathrm{H}_{2} \mathrm{~S}$ on restenosis after BA. This discovery may lead to potential novel therapies, which can improve the prognosis of PAD patients after interventional treatments.

\section{Acknowledgements}

This study was supported by grants from the Capital Medical Development Scientific Research Foundation of China (2007-2047), and the Scientific Research Foundation of Peking University Ninth Clinical Hospital, Beijing, China (2007-07).

\section{References}

1. Kasapis C, Henke PK, Chetcuti SJ, et al: Routine stent implantation vs. percutaneous transluminal angioplasty in femoropopliteal artery disease: a meta-analysis of randomized controlled trials. Eur Heart J 30: 44-55, 2009.

2. Dzau VJ, Braun-Dullaeus RC and Sedding DG: Vascular proliferation and atherosclerosis: new perspectives and therapeutic strategies. Nat Med 8: 1249-1256, 2002.

3. Wang R: Two's company, three's a crowd: $\mathrm{Can}_{2} \mathrm{H}_{2} \mathrm{~S}$ be the third endogenous gaseous transmitter? FASEB J 16: 1792-1798, 2002.

4. Tang C, Li X and Du J: Hydrogen sulfide as a new endogenous gaseous transmitter in the cardiovascular system. Curr Vasc Pharmacol 4: 17-22, 2006.

5. Du J, Zhang C, Yan $\mathrm{H}$ and Tang C: A newly found gasotransmitter, hydrogen sulfide, in the pathogenesis of hypertension and other cardiovascular diseases. Curr Hypertens Rev 2: 123-126, 2006.

6. Lefer DJ: A new gaseous signaling molecule emerges: cardioprotective role of hydrogen sulfide. Proc Natl Acad Sci USA 104 17907-17908, 2007.
7. Jiang HL, Wu HC, Li ZL, Geng B and Tang CS: Changes of the new gaseous transmitter $\mathrm{H}_{2} \mathrm{~S}$ in patients with coronary heart disease. J First Mil Med Univ 25: 951-954, 2005 (In Chinese).

8. Wang Y, Zhao X, Jin H, Wei H, Li W, Bu D, Tang X, Ren Y, Tang $\mathrm{C}$ and Du J: Role of hydrogen sulfide in the development of atherosclerotic lesions in apolipoprotein $\mathrm{E}$ knockout mice. Arterioscler Thromb Vasc Biol 29: 173-179, 2009.

9. Yan H, Du J and Tang C: The possible role of hydrogen sulfide on the pathogenesis of spontaneous hypertension in rats. Biochem Biophys Res Commun 313: 22-27, 2004.

10. Durand E, Mallat Z, Addad F, Vilde F, Desnos M, Guérot C, Tedgui A and Lafont A: Time courses of apoptosis and cell proliferation and their relationship to arterial remodeling and restenosis after angioplasty in an atherosclerotic rabbit model J Am Coll Cardiol 39: 1680-1685, 2002.

11. LeVeen RF, Wolf GL and Villanueva TG: New rabbit atherosclerosis model for the investigation of transluminal angioplasty. Invest Radiol 17: 470-475, 1982.

12. Brasselet C, Durand E, Addad F, et al: Collagen and elastin crosslinking: a mechanism of constrictive remodeling after arterial injury. Am J Physiol Heart Circ Physiol 289: H2228-H2233, 2005.

13. Saussoy P, Vaerman JL, Straetmans N, Deneys V, Cornu G, Ferrant A and Latinne D: Differentiation of acute myeloid leukemia from B- and T-lineage acute lymphoid leukemias by real-time quantitative reverse transcription-PCR of lineage marker mRNAs. Clin Chem 50: 1165-1173, 2004.

14. Zhou Z, Lauer MA, Wang K, et al: Effect of anti-tumor necrosis factor-alpha polyclonal antibody on restenosis after balloon angioplasty in a rabbit atherosclerotic model. Atherosclerosis 161: $153-159,2002$.

15. Bromley M, Rew D, Becciolini A, Balzi M, Chadwick C, Hewitt D, Li YQ and Potten CS: A comparison of proliferation markers (BrdUrd, Ki-67, PCNA) determined at each cell position in the crypts of normal human colonic mucosa. Eur J Histochem 40: 89-100, 1996.

16. Thompson GA, Datko AH and Mudd SH: Methionine synthesis in lemna: inhibition of cystathionine gamma-synthase by propargylglycine. Plant Physiol 70: 1347-1352, 1982.

17. Yang G, Cao K, Wu L and Wang R: Cystathionine gamma-lyase overexpression inhibits cell proliferation via a $\mathrm{H}_{2} \mathrm{~S}$-dependent modulation of $\mathrm{ERK}_{1 / 2}$ phosphorylation and $\mathrm{p} 21^{\mathrm{Cip} / \mathrm{WAK}-\mathrm{I}}$. J Biol Chem 279: 49199-49205, 2004.

18. Yang G, Wu L and Wang R: Pro-apoptotic effect of endogenous $\mathrm{H}_{2} \mathrm{~S}$ on human aorta smooth muscle cells. FASEB J 20: 553-555, 2006.

19. Baskar R, Sparatore A, Del Soldato P and Moore PK: Effect of S-diclofenac, a novel hydrogen sulfide releasing derivative inhibit rat vascular smooth muscle cell proliferation. Eur J Pharmacol 594: 1-8, 2008.

20. Blankenberg S, Barbaux S and Tiret L: Adhesion molecules and atherosclerosis. Atherosclerosis 170: 191-203, 2003.

21. Ross R: Atherosclerosis-an inflammatory disease. N Engl J Med 340: 115-126, 1999. 